\title{
Critical behaviour of (2+1)-dimensional QED: 1/N-corrections
}

\author{
Anatoly V. Kotikov ${ }^{1, \star}$ and Sofian Teber ${ }^{2,3, \star \star}$ \\ ${ }^{1}$ Bogoliubov Laboratory of Theoretical Physics, Joint Institute for Nuclear Research, 141980 Dubna, Russia \\ ${ }^{2}$ Sorbonne Universités, UPMC Univ Paris 06, UMR 7589, LPTHE, F-75005, Paris, France and CNRS, UMR \\ 7589, LPTHE, F-75005, Paris, France \\ ${ }^{3}$ CNRS, UMR 7589, LPTHE, F-75005, Paris, France
}

\begin{abstract}
We present recently obtained results for dynamical chiral symmetry breaking studied within $(2+1)$-dimensional QED with $N$ four-component fermions. The leading and next-to-leading orders of the $1 / N$ expansion are computed exactly in an arbitrary non-local gauge.
\end{abstract}

\section{Introduction}

In these Proceedings we present the results of our recent papers [1, 2], where the critical behavior of Quantum Electrodynamics in $2+1$ dimensions $\left(\mathrm{QED}_{3}\right)$ has been studied. $\mathrm{QED}_{3}$ is described by the Lagrangian:

$$
L=\bar{\Psi}(i \hat{\partial}-e \hat{A}) \Psi-\frac{1}{4} F_{\mu \nu}^{2},
$$

where $\Psi$ is taken to be a four component complex spinor. In the presence of $N$ fermion flavours, the model has a $U(2 N)$ symmetry. A (parity-invariant) fermion mass term, $m \bar{\Psi} \Psi$, breaks this symmetry to $U(N) \times U(N)$. In the massless case, loop expansions are plagued by infrared divergences. The latter soften upon analyzing the model in a $1 / N$ expansion [3]. Since the theory is super-renormalizable, the mass scale is then given by the dimensionless coupling constant: $a=N e^{2} / 8$, which is kept fixed as $N \rightarrow \infty$. Early studies of this model $[4,5]$ suggested that the physics is rapidly damped at momentum scales $p \gg a$ and that a fermion mass term breaking the flavour symmetry is dynamically generated at scales which are orders of magnitude smaller than the intrinsic scale $a$. Since then, dynamical chiral symmetry breaking $\left(\mathrm{D}_{\chi} \mathrm{SB}\right)$ in $\mathrm{QED}_{3}$ and the dependence of the dynamical fermion mass on $N$ have been the subject of extensive studies, see, e.g., [1, 2, 4-13].

One of the central issue is related to the value of the critical fermion number, $N_{c}$, which is such that $\mathrm{D} \chi \mathrm{SB}$ takes place only for $N<N_{c}$. An accurate determination of $N_{c}$ is of crucial importance to understand the phase structure of $\mathrm{QED}_{3}$ with far reaching implications from particle physics to planar condensed matter physics systems having relativistic-like low-energy excitations [14]. It turns out that the values that can be found in the literature vary from $N_{c} \rightarrow \infty[4,6]$ corresponding to $\mathrm{D} \chi \mathrm{SB}$ for all values of $N$, all the way to $N_{c} \rightarrow 0$ in the case where no sign of $\mathrm{D} \chi \mathrm{SB}$ is found [7].

\footnotetext{
${ }^{\star}$ e-mail: kotikov@theor.jinr.ru

$\star \star$ e-mail: teber@1pthe.jussieu.fr
} 


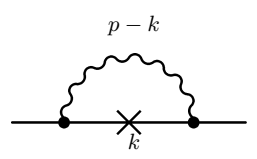

Figure 1. LO diagram to the dynamically generated mass $\Sigma(p)$. The crossed line denotes mass insertion.

Of importance to us in the following, is the approach of Appelquist et al. [5] who found that $N_{c}=32 / \pi^{2} \approx 3.24$ by solving the Schwinger-Dyson (SD) gap equation using a leading order (LO) $1 / N$-expansion. Lattice simulations in agreement with a finite non-zero value of $N_{c}$ can be found in [8]. Soon after the analysis of [5], Nash approximately included next-to-leading order (NLO) corrections and performed a partial resummation of the wave-function renormalization constant at the level of the gap equation; he found [9]: $N_{c} \approx 3.28$.

Recently, in [1], the NLO corrections could be computed exactly in the Landau gauge upon refining the analysis of [10]. This led to $N_{c} \approx 3.29$, a value which is surprisingly close to the one of Nash in [9]. More recently, in [2], the results of [1] were generalized to an arbitrary non-local gauge [15]. Moreover, it was shown in [2] that a resummation of the wave-function renormalization yields a strong suppression of the gauge dependence of the critical fermion flavour number, $N_{c}(\xi)$ where $\xi$ is the gauge fixing parameter, which is such that $\mathrm{D} \chi \mathrm{SB}$ takes place for $N<N_{c}(\xi)$. Neglecting the gauge-dependent terms yields $N_{c}=2.8469$, that coincides with results in [11]. In the general case, it is found that: $N_{c}(1)=3.0084$ in the Feynman gauge, $N_{c}(0)=3.0844$ in the Landau gauge and $N_{c}(2 / 3)=3.0377$ in the $\xi=2 / 3$ gauge where the leading order fermion wave function is finite. These results suggest that $\mathrm{D} \chi \mathrm{SB}$ should take place for integer values $N \leq 3$. Using a very different method, Herbut obtained [12] a close value: $N_{c} \approx 2.89$.

It is the purpose of this work to review some of the basic steps of papers [1,2] which represent an essential improvement with respect to Nash's approximate NLO results derived some 30 years ago.

\section{Schwinger-Dyson equations}

With the conventions of [1], the inverse fermion propagator is defined as: $S^{-1}(p)=[1+$ $A(p)](i \hat{p}+\Sigma(p))$ where $A(p)$ is the fermion wave function and $\Sigma(p)$ is the dynamically generated parity-conserving mass which is taken to be the same for all the fermions. The SD equation for the fermion propagator may be decomposed into scalar and vector components as follows:

$$
\tilde{\Sigma}(p)=\frac{2 a}{N} \operatorname{Tr} \int \frac{d^{3} k}{(2 \pi)^{3}} \frac{\gamma^{\mu} D_{\mu v}(p-k) \Sigma(k) \Gamma^{v}(p, k)}{[1+A(k)]\left(k^{2}+\Sigma^{2}(k)\right)}, A(p) p^{2}=-\frac{2 a}{N} \operatorname{Tr} \int \frac{d^{3} k}{(2 \pi)^{3}} \frac{D_{\mu v}(p-k) \hat{p} \gamma^{\mu} \hat{k} \Gamma^{v}(p, k)}{[1+A(k)]\left(k^{2}+\Sigma^{2}(k)\right)},
$$

where $\tilde{\Sigma}(p)=\Sigma(p)[1+A(p)], D_{\mu v}(p)$ is the photon propagator in the non-local $\xi$-gauge:

$$
D_{\mu \nu}(p)=\frac{P_{\mu \nu}^{\xi}(p)}{p^{2}[1+\Pi(p)]}, \quad P_{\mu \nu}^{\xi}(p)=g_{\mu \nu}-(1-\xi) \frac{p_{\mu} p_{v}}{p^{2}},
$$

$\Pi(p)$ is the polarization operator and $\Gamma^{\gamma}(p, k)$ is the vertex function. In the following, (2) will be studied for an arbitrary value of the gauge-fixing parameter $\xi$. All calculations will be performed with the help of the standard rules of perturbation theory for massless Feynman diagrams as in [16], see also the recent short review [17]. For the most complicated diagrams, the Gegenbauer polynomial technique will be used following [18]. 


\section{Gap equation at leading order}

The LO approximations in the $1 / N$ expansion are given by: $A(p)=0, \Pi(p)=a /|p|$ and $\Gamma^{\nu}(p, k)=\gamma^{v}$, where the fermion mass has been neglected in the calculation of $\Pi(p)$. A single diagram contributes to the gap equation (2) at LO, see figure 1, and the latter reads:

$$
\Sigma(p)=\frac{8(2+\xi) a}{N} \int \frac{d^{3} k}{(2 \pi)^{3}} \frac{\Sigma(k)}{\left(k^{2}+\Sigma^{2}(k)\right)\left[(p-k)^{2}+a|p-k|\right]} .
$$

Following [5], we consider the limit of large $a$ and linearize (4) which yields:

$$
\Sigma(p)=\frac{8(2+\xi)}{N} \int \frac{d^{3} k}{(2 \pi)^{3}} \frac{\Sigma(k)}{k^{2}|p-k|} .
$$

The mass function may then be parameterized as [5]: $\Sigma(k)=B\left(k^{2}\right)^{-\alpha}$, where $B$ is arbitrary and the index $\alpha$ has to be self-consistently determined. Using this Ansatz, (5) leads to the LO gap equation: $\left(\beta^{-1}=\alpha(1 / 2-\alpha)\right.$ and $\left.L \equiv \pi^{2} N\right)$

$$
1=\frac{(2+\xi) \beta}{L} \text { and } \alpha_{ \pm}=\frac{1}{4}\left(1 \pm \sqrt{1-\frac{16(2+\xi)}{L}}\right),
$$

which reproduces the solution given by Appelquist et al. [5]. The gauge-dependent critical number of fermions: $N_{c} \equiv N_{c}(\xi)=16(2+\xi) / \pi^{2}$, is such that $\Sigma(p)=0$ for $N>N_{c}$ and $\Sigma(0) \simeq \exp \left[-2 \pi /\left(N_{c} / N-\right.\right.$ $1)^{1 / 2}$ ], for $N<N_{c}$. Thus, D $\chi \mathrm{SB}$ occurs when $\alpha$ becomes complex, that is for $N<N_{c}$.

The gauge-dependent fermion wave function may be computed in a similar way. At LO, (2) simplifies as:

$$
A(p) p^{2}=-\frac{2 a}{N} \operatorname{Tr} \int \frac{d^{D} k}{(2 \pi)^{D}} \frac{P_{\mu \nu}^{\xi}(p-k) \hat{p} \gamma^{\mu} \hat{k} \gamma^{v}}{k^{2}|p-k|},
$$

where the integral has been dimensionally regularized with $D=3-2 \varepsilon$. Taking the trace and computing the integral on the r.h.s. yields:

$$
A(p)=\frac{\bar{\mu}^{2 \varepsilon}}{p^{2 \varepsilon}} C_{1}(\xi)+\mathrm{O}(\varepsilon), C_{1}(\xi)=+\frac{2}{3 \pi^{2} N}\left((2-3 \xi)\left[\frac{1}{\varepsilon}-2 \ln 2\right]+\frac{14}{3}-6 \xi\right),
$$

where the $\overline{M S}$ parameter $\bar{\mu}$ has the standard form $\bar{\mu}^{2}=4 \pi e^{-\gamma_{E}} \mu^{2}$ with the Euler constant $\gamma_{E}$. We note that in the $\xi=2 / 3$-gauge, the value of $A(p)$ is finite and $C_{1}(\xi=2 / 3)=+4 /\left(9 \pi^{2} N\right)$. From (8), the LO wave-function renormalization constant may be extracted: $\lambda_{A}=\mu(d / d \mu) A(p)=4(2-3 \xi) /\left(3 \pi^{2} N\right)$ a result which coincides with the one of [19].

\section{Next-to-leading order}

We now consider the NLO contributions and parametrize them as:

$$
\Sigma^{(\mathrm{NLO})}(p)=\left(\frac{8}{N}\right)^{2} B \frac{\left(p^{2}\right)^{-\alpha}}{(4 \pi)^{3}}\left(\Sigma_{A}+\Sigma_{1}+2 \Sigma_{2}+\Sigma_{3}\right),
$$

where each contribution to the linearized gap equation is represented graphically in figure 2 . The gap equation has the following general form:

$$
1=\frac{(2+\xi) \beta}{L}+\frac{\bar{\Sigma}_{A}(\xi)+\bar{\Sigma}_{1}(\xi)+2 \bar{\Sigma}_{2}(\xi)+\bar{\Sigma}_{3}(\xi)}{L^{2}}, \quad \bar{\Sigma}_{i}=\pi \Sigma_{i}, \quad(i=1,2,3 . A)
$$




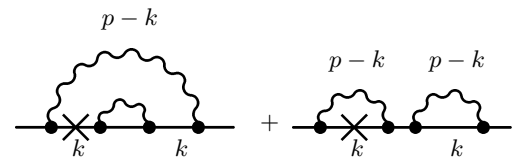

A)

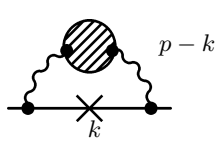

1)

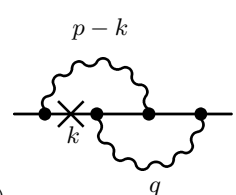

2)

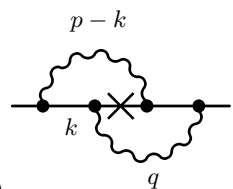

3)

Figure 2. NLO diagrams to the dynamically generated mass $\Sigma(p)$. The shaded blob defines the two-loop polarization operator, see $[1,2]$ for details.

Performing the calculation of the diagrams shown in figure 2 (see [1, 2]), the gap equation (10) may be written in an explicit form as:

$$
1=\frac{(2+\xi) \beta}{L}+\frac{1}{L^{2}}\left[8 S(\alpha, \xi)-2(2+\xi) \hat{\Pi} \beta+\left(-\frac{5}{3}+\frac{26}{3} \xi-3 \xi^{2}\right) \beta^{2}-8 \beta\left(\frac{2}{3}(1-\xi)-\xi^{2}\right)\right],
$$

where $\hat{\Pi}=92 / 9-\pi^{2}$ arises from the two-loop polarization operator in dimension $D=3[20,21]$ and $S(\alpha, \xi)$ contains the contributions of complicated diagrams. Considering (11) directly at the critical point $\alpha=1 / 4$, i.e., at $\beta=16$, we have

$$
L_{c}^{2}-16(2+\xi) L_{c}-8\left[S(\xi)-4(2+\xi) \hat{\Pi}-16\left(4-50 \xi / 3+5 \xi^{2}\right)\right]=0,
$$

where $S(\xi)=S(\alpha=1 / 4, \xi)$ and

$8 S(\xi)=8(1-\xi) R_{1}+\left(\xi^{2}-1\right) R_{2}-\left(7+16 \xi-3 \xi^{2}\right) \frac{P_{2}}{16}, R_{1}=163.7428, \quad R_{2}=209.175, \quad P_{2}=1260.720$

Solving (12), we have two standard solutions:

$$
L_{c, \pm}=8(2+\xi) \pm \sqrt{d_{1}(\xi)}, \quad d_{1}(\xi)=8\left[S(\xi)-8\left(4-\frac{112}{3} \xi+9 \xi^{2}+\frac{2+\xi}{2} \hat{\Pi}\right)\right]
$$

Combining these values with the one of $\hat{\Pi}$, yields: $N_{c}(\xi=0)=3.29, N_{c}(\xi=2 / 3)=3.09$, where "-" solutions are unphysical and there is no solution in the Feynman gauge. The range of $\xi$-values for which there is a solution corresponds to $\xi_{-} \leq \xi \leq \xi_{+}$, where $\xi_{+}=0.88$ and $\xi_{-}=-2.36$.

\section{Resummation}

Following [9], we would like to resum the LO term together with part of the NLO corrections containing terms $\sim \beta^{2}$. In order to do so, we will now rewrite the gap equation (11) in a form which is suitable for resummation. This amounts to extract the terms $\sim \beta$ and $\sim \beta^{2}$ from the complicated part of the fermion self-energy, $S(\alpha, \xi)$, yielding:

$$
S(\alpha, \xi)=\frac{1}{4}(1-\xi) \beta(3 \beta-8)-\frac{1}{2} \xi(4+\xi) \beta+\tilde{S}(\alpha, \xi) .
$$

At the critical point $\alpha=1 / 4(\beta=16), \tilde{S}(\xi)=\tilde{S}(\alpha=1 / 4, \xi)$ has the following form:

$$
8 \tilde{S}(\xi)=8(1-\xi) \tilde{R}_{1}+\left(\xi^{2}-1\right) \tilde{R}_{2}-\left(7+16 \xi-3 \xi^{2}\right) \frac{\tilde{P}_{2}}{16}, \quad \tilde{R}_{1}=3.7428, \quad \tilde{R}_{2}=1.175, \quad \tilde{P}_{2}=-19.28
$$


With the help of the results (16), the gap equation (11) may be written as:

$$
1=\frac{(2+\xi) \beta}{L}+\frac{1}{L^{2}}\left[8 \tilde{S}(\alpha, \xi)-2(2+\xi) \hat{\Pi} \beta+\left(\frac{2}{3}-\xi\right)(2+\xi) \beta^{2}+4 \beta\left(\xi^{2}-\frac{4}{3} \xi-\frac{16}{3}\right)\right] .
$$

At this point (11) and (17) are strictly equivalent to each other and yield the same values for $N_{c}(\xi)$. Equation (17) is the convenient starting point to perform a resummation of the wave function renormalization constant. To do it (see details in [2]) (17) can now be expressed as:

$$
1=\frac{8 \beta}{3 L}+\frac{1}{L^{2}}\left[8 \tilde{S}(\alpha, \xi)-\frac{16}{3} \beta\left(\frac{40}{9}+\hat{\Pi}\right)\right],
$$

which displays a strong suppression of the gauge dependence even at NLO as $\xi$-dependent terms do exist but they enter the gap equation only through the rest, $\tilde{S}$, which is very small numerically.

We now consider (18) at the critical point, $\alpha=1 / 4(\beta=16)$, which yields:

$$
L_{c}^{2}-\frac{128}{3} L_{c}-\left[8 \tilde{S}(\xi)-\frac{256}{3}\left(\frac{40}{9}+\hat{\Pi}\right)\right]=0 .
$$

Solving (19), we have two standard solutions:

$$
L_{c, \pm}=\frac{64}{3} \pm \sqrt{d_{2}(\xi)}, \quad d_{2}(\xi)=\left(\frac{64}{3}\right)^{2}+\left[8 \tilde{S}(\xi)-\frac{256}{3}\left(\frac{40}{9}+\hat{\Pi}\right)\right] .
$$

In order to provide a numerical estimate for $N_{c}$, we have used the values of $\tilde{R}_{1}, \tilde{R}_{2}$ and $\tilde{P}_{2}$ of (16). Combining these values together with the value of $\hat{\Pi}$, yields, for $N_{c}(\xi)$ ("-" solutions being unphysical):

$$
N_{c}(0)=3.08, \quad N_{c}(2 / 3)=3.04, \quad N_{c}(1)=3.01 .
$$

Actually, solutions exist for a broad range of values of $\xi$ : $\tilde{\xi}_{-} \leq \xi \leq \tilde{\xi}_{+}$, where $\tilde{\xi}_{+}=4.042$ and $\tilde{\xi}_{-}=-8.412$; this is consistent with the weak $\xi$-dependence of the gap equation. Moreover, following [22], we think that the "right(est)" gauge choice is one close to $\xi=2 / 3$ where the LO fermion wave function is finite. Indeed, upon resumming the theory, the value of $N_{c}(\xi)$ increases (decreases) for small (large) values of $\xi$. For $\xi=2 / 3$, the value of $N_{c}$ is very stable, decreasing only by $1-2 \%$ during resummation. Finally, if we neglect the rest, i.e., $\tilde{S}(\xi)=0$ in (19), the gap equation becomes $\xi$-independent and we have: $\bar{L}_{c}=28.0981$ and therefore: $\bar{N}_{c}=2.85$, a value that coincides with the one in [11].

\section{Conclusion}

We have presented the studies $[1,2]$ of $\mathrm{D} \chi \mathrm{SB}$ in $\mathrm{QED}_{3}$ by including $1 / N^{2}$ corrections to the SD equation exactly and taking into account the full $\xi$-dependence of the gap equation. Following Nash, the wave function renormalization constant has been resummed at the level of the gap equation leading to a very weak gauge-variance of the critical fermion number $N_{c}$. The value obtained for the latter, (21), suggests that $\mathrm{D} \chi \mathrm{SB}$ takes place for integer values $N \leq 3$ in $\mathrm{QED}_{3}$.

Notice that the large- $N$ limit of the photon propagator in $\mathrm{QED}_{3}$ has precisely the same momentum dependence as the one in the so-called reduced QED, see [22]. One difference is that the gauge fixing parameter in reduced QED is twice less than the one in $\mathrm{QED}_{3}$. Such a difference can be taken into account with the help of our present results for $\mathrm{QED}_{3}$ together with the multi-loop results obtained in $[20,23]$. The case of reduced QED, and its relation with dynamical gap generation in graphene which is the subject of active ongoing research, see, e.g., the reviews [24], was considered in our paper [25]. 


\section{References}

[1] A. V. Kotikov, V. I. Shilin and S. Teber, Phys. Rev. D 94, 056009 (2016)

[2] A. V. Kotikov and S. Teber, arXiv:1609.06912 [hep-th]

[3] T. Appelquist and R. Pisarski, Phys. Rev. D 23, 2305 (1981). R. Jackiw and S. Templeton, Phys. Rev. D 23, 2291 (1981). T. Appelquist and U. Heinz, Phys. Rev. D 24, 2169 (1981)

[4] R. Pisarski, Phys. Rev. D 29, 2423 (1984)

[5] T. Appelquist, D. Nash and L. C. R. Wijewardhana, Phys. Rev. Lett. 60, 2575 (1988)

[6] M. R. Pennington and D. Walsh, Phys. Lett. 253B, 246 (1991). D. C. Curtis, M. R. Pennington and D. Walsh, Phys. Lett. 295B, 313 (1992). R. Pisarski, Phys. Rev. D 44, 1866 (1991). V. Azcoiti and X. Q. Luo, Nucl. Phys. Proc. Suppl. 30, 741 (1993). V. Azcoiti, V. Laliena and X. Q. Luo, Nucl. Phys. Proc. Suppl. 47, 565 (1996)

[7] D. Atkinson, P. W. Johnson and P. Maris, Phys. Rev. D 42, 602 (1990). N. Karthik and R. Narayanan, Phys. Rev. D 93, 045020 (2016). Phys. Rev. D 94, 065026 (2016)

[8] E. Dagotto, A. Kocic and J. B. Kogut, Phys. Rev. Lett. 62, 1083 (1989). Nucl. Phys. B 334, 279 (1990). S. J. Hands, J. B. Kogut, L. Scorzato and C. G. Strouthos, Phys. Rev. B 70, 104501 (2004). C. Strouthos and J. B. Kogut, PoS (LAT 2007) 278 (2007)

[9] D. Nash, Phys. Rev. Lett. 62, 3024 (1989)

[10] A. V. Kotikov, JETP Lett. 58, 734 (1993). Phys. Atom. Nucl. 75, 890 (2012)

[11] V. P. Gusynin and P. K. Pyatkovskiy, arXiv:1607.08582 [hep-ph]

[12] I. F. Herbut, Phys. Rev. D 94, 025036 (2016)

[13] A. Bashir, A. Raya, S. Sanchez-Madrigal and C. D. Roberts, Few Body Syst. 46, 229 (2009)

[14] J. B. Marston and I. Affleck, Phys. Rev. B 16, 11538 (1989). L. B. Ioffe and A. I. Larkin, Phys. Rev. B 13, 8988 (1989). G. W. Semenoff, Phys. Rev. Lett. 53, 2449 (1984). P. R. Wallace, Phys. Rev. 71, 622 (1947)

[15] E. H. Simmons, Phys. Rev. D 42, 2933 (1990). T. Kugo and M. G. Mitchard, Phys. Lett. B 282, $162(1992)$

[16] D. I. Kazakov, Phys. Lett. 133B, 406 (1983). "Analytical Methods For Multiloop Calculations: Two Lectures On The Method Of Uniqueness" - 1984. Preprint JINR E2-84-410, Dubna

[17] S. Teber and A. V. Kotikov, arXiv:1602.01962 [hep-th]

[18] A. V. Kotikov, Phys. Lett. B 375, 240 (1996)

[19] J. A. Gracey, Nucl. Phys. B 414, 614 (1994)

[20] S. Teber, Phys. Rev. D 86, 025005 (2012). A. V. Kotikov and S. Teber, Phys. Rev. D 87, 087701 (2013)

[21] V. P. Gusynin, A. H. Hams and M. Reenders, Phys. Rev. D 63, 045025 (2001). J.A. Gracey, Phys. Lett. B 317, 415 (1993)

[22] E. V. Gorbar, V. P. Gusynin and V. P. Miransky, Phys. Rev. D 64, 105028 (2001). E. C. Marino, Nucl. Phys. B 408, 551 (1993). N. Dorey and N. E. Mavromatos, Nucl. Phys. B 386, 614 (1992).

A. Kovner and B. Rosenstein, Phys. Rev. D 42, 4748 (1990)

[23] A. V. Kotikov and S. Teber, Phys. Rev. D 89, 065038 (2014). S. Teber, Phys. Rev. D 89, 067702 (2014)

[24] V. N. Kotov, B. Uchoa, V. M. Pereira, F. Guinea and A. H. Castro Neto, Rev. Mod. Rev. 84, 1067 (2012). V. A. Miransky and I. A. Shovkovy, Phys. Rept. 576, 1 (2015). V. P. Gusynin, Prob. Atomic Sci. Technol. 2013N3, 29 (2013)

[25] A. V. Kotikov and S. Teber, arXiv:1610.00934 [hep-th] 